\title{
Safe and securely guided tracheal puncture in percutaneous dilatational tracheotomy in critical ill patients: case report
}

\begin{abstract}
This article reports on 2 cases of critical ill patients in need of tracheotomy. Both patients were subjects to percutaneous dilatational tracheotomy but had a difficult neck anatomy and hence difficult to find the proper puncture site that should be between the 2nd and 3rd or 3rd and 4th tracheal rings. To make it possible, the punctures were guided with the help of a new device - SafeTrach. This device has an inner and an outer shank that functions like a tweezers. When the inner shank is in place inside the orotracheal tube and the outer shank is connected to the inner shank, the puncture needle in the tip of the outer shank is pointing against the tip of the inner shank inside the trachea. This part of the inner shank is protruding from the tube, ensuring that the puncture will not be made in the tube or in the posterior wall of the trachea and in a proper angle. In both cases the procedures were successful and made in less than 10 minutes.
\end{abstract}

Keywords: tracheotomy, tracheostomy, percutaneous, dilatation, tracheostoma; intensive care
Volume 10 Issue I - 2018

\author{
Gregori Margolin,' Johan Ullman, ${ }^{2}$ Jonas \\ Karling ${ }^{3}$ \\ 'Department of Otolaryngology and Head \& Neck Surgery \\ and Department of Oncology, Karolinska University Hospital, \\ Sweden \\ 2Department of Anesthesiology and Intensive Care, Karolinska \\ University Hospital, Sweden \\ ${ }^{3}$ Department of Otolaryngology and Head \& Neck Surgery, \\ Karolinska University Hospital, Sweden
}

\begin{abstract}
Correspondence: Jonas Karling, Department of Otolaryngology and Head \& Neck Surgery, Karolinska University Hospital, Hoglandstorget 7, 1677 I Bromma, Sweden Tel +467075540 66, Email jonkar@me.com
\end{abstract}

Received: February 07, 2018 | Published: February 15, 2018
Abbreviations: PDT, percutaneous dilatational tracheotomy; ECMO, extracorporeal membrane oxygenation

\section{Introduction}

Patients who require intensive care often need tracheotomy to aid weaning from assisted ventilation. PDT has proved to be an efficient and cost effective choice instead of conventional open surgical tracheotomy. However, when the patients ' anatomy is disadvantageous many physicians experience the PDT-technique unsafe and difficult to learn. Dempsey et al reported intraoperative technical difficulties in $26 \%$ of their cases. ${ }^{1}$ According to Rosseland et al., ${ }^{2}$ surgery related complications in conjunction with disadvantageous anatomy were found in $17.5 \%$ of their cases. The most complicated and critical step in PDT is the puncturing procedure. Some of the difficulties doctors experience are:

a. Difficulties in identifying the site for tracheal puncture.

b. Risk of having the puncturing needle hit into the orotracheal tube

c. The orotracheal tube has to be pulled back with risk of extubation.

d. Risk of penetrating too deeply injuring the posterior tracheal wall or to be outside the trachea.

e. Risk of injuring the fiber optic bronchoscope.

The new invention, SafeTrach, addresses all these problems. This device has an inner and an outer shank that, when combined functions like a tweezers (Figure 1-4). The inner shank is introduced through the orotracheal tube and when the outer shank has been connected to the inner shank the puncture needle will be guided to a correct puncture. ${ }^{3,4}$

\section{Case presentations}

\section{Case no}

A 56years old female patient treated twice for tonsil cancer and later cancer of the tongue. She had had radiotherapy twice for both those cancers. She had also gone through a neck dissection. She developed adverse event such as osto-radionecrosis of the mandible and pathological fracture, infection and fistula. She was planned for reconstruction with a free fibula flap and because of that she needed a tracheotomy in order to guarantee free airways. To minimize the risk of infection when performing two surgical procedures close to each other, PDT is preferable and was the tracheotomy of choice for this patient. The patient was intubated with an orotracheal tube size 7 . The ventilation was interrupted shortly for introducing the SafeTrach inner shank through the tube. The ventilation was then immediately restored through the inner shank and remained so during the entire procedure. The site for the puncture was decided and an incision through the skin was made. The outer shank was connected to the inner shank and the puncture needle pointed one centimeter below the incision why the whole package including the inner shaft and the orotracheal tube was withdrawn one centimeter until the orotracheal cuff hit against the vocal cords and the puncture needle pointed to the incision. Then the needle was pressed through the trachea and bubble in a syringe connected to the needle confirmed proper access to the tracheal lumen. A guide wire was then lead through the needle down into trachea and a tracheostoma was created with a Ciaglia dilatator. The patient was ventilated via the inner shaft and the orotracheal tube until the tracheal cannula was insufflated. The procedure lasted for 10minutes.

\section{Case no 2}

A 73 years old male patient treated with implant of mitral valve due to chordaruptur. Postoperative complications with kidney insufficiency, infected sternotomy, pneumonia and heart failure. The patient was treated with ECMO and hemodialysis and was in need of tracheotomy because of extended need of ventilator support. He was obese $(120 \mathrm{~kg})$. At the thoracic clinic PDT is preferable before open surgery due to the narrow distance to the sternum and in this case the yet open chest. The PDT was made in the same way as described for case no 1 but this patient had an orotracheal tube size 8 and the PDT was made bedside. 


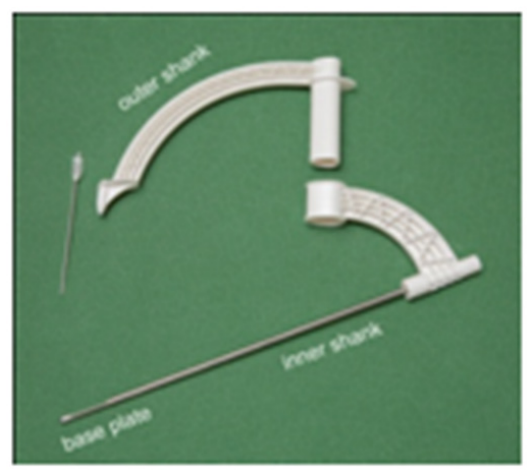

Figure I The SafeTrach device unassembled.

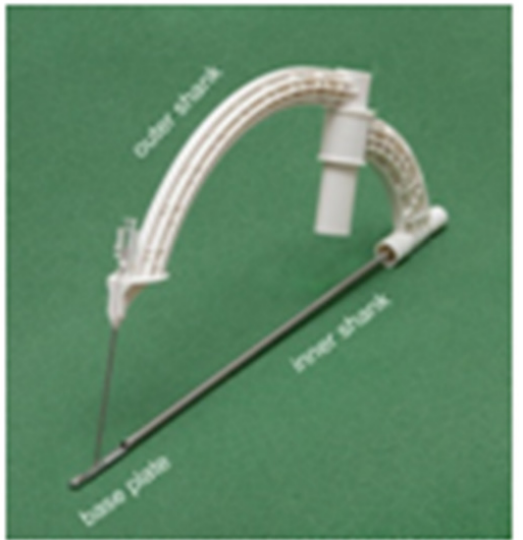

Figure 2 The SafeTrach device assembled.

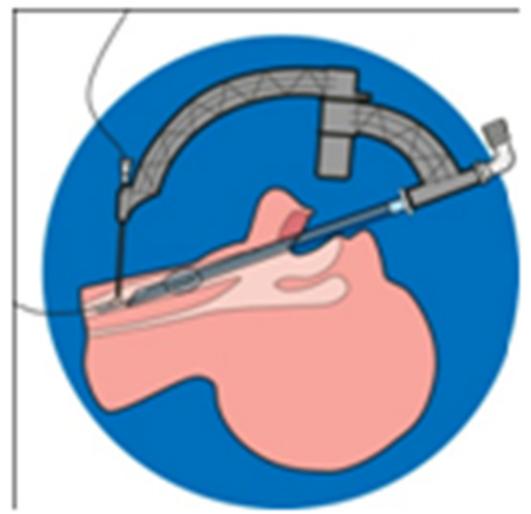

Figure 3 The instrument has guided the needle to a proper puncture.

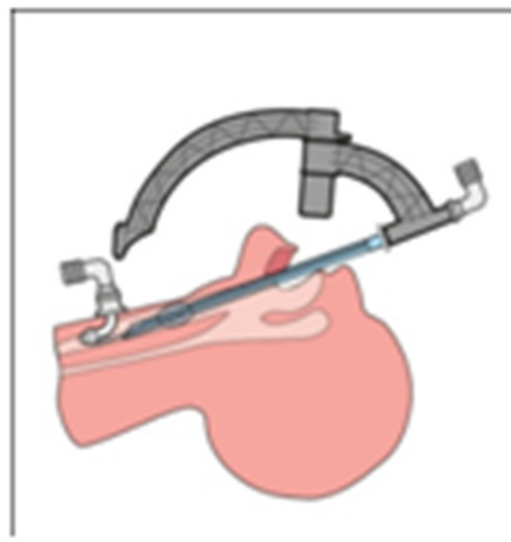

Figure 4 In the final part of the procedure there are two secure respiratory pathways: a) through the inner shaft and b) through the tracheal cannula. When the cannula is cuffed, the ventilation is moved from the inner shaft to the cannula.
Due to the obesity it was difficult to palpate the structures of the neck to find the right position for the puncture. In order to reach a proper initial position, the orotracheal tube was retracted under laryngoscopic overview until the tube cuff reached the vocal cords. Then the SafeTrach inner shaft was introduced through the orotracheal tube but the tube with the inner shaft had still to be retracted about one centimeter until the needle pointed to a proper puncture site. Then the puncture and dilatation went well and the inner shaft and tube was removed when the tracheal cannula was in place and the cuff insufflated.

\section{Discussion}

It is often difficult to locate the anatomical structures of the neck by palpation. This is especially difficult in women who have less prominent thyroid cartilage. This is also the case if the neck is short or thick and swollen. ${ }^{1,2}$ It is very important not to puncture the posterior tracheal wall and/or the orotracheal tube. The aim is also to make the puncture as central as possible in the middle of trachea and preferably between the $2^{\text {nd }}$ and $3^{\text {rd }}$ or $3^{\text {rd }}$ and $4^{\text {th }}$ tracheal rings.

In both patients that were included in this case report, the anatomic conditions were extremely difficult and not at first glance possible objects for bedside PDT. However, the strong incitement to avoid an open conventional tracheotomy (risk for infection and bleeding) made the decision to try SafeTrach PDT.

With the aid of the SafeTrach device with it's tweezers like construction it was possible to locate the position for the puncture as the device gives guidance to how many centimeters below the vocal cords the puncture site will be. Furthermore, the device functions as a three-dimensional guide when making the puncture and has both an inner and an outer stop to prevent too deep puncture. The device guides furthermore the needle below the tip of the orotracheal tube and in the middle of the tracheal lumen. Another advantage with this system is that the patient is ventilated through the inner shank and the tube during the entire procedure all the way until the tracheal cannula is securely in place and cuffed.

\section{Acknowledgments}

None.

\section{Conflicts of interest}

Gregori Margolin and Jonas Karling are inventors of SafeTrach and shareholders in company SafeTrach AB.

\section{Sponsorships}

None.

\section{Funding source}

None.

\section{References}

1. Dempsey GA, Grant CA, Jones TM. Percutaneous tracheostomy: a 6 yr prospective evaluation of the single tapered dilator technique. $\mathrm{Br} J$ Anaesth. 2010;105(6):782-788.

2. Rosseland LA, Laake JH, Stubhaug A. Percutaneous dilatational tracheotomy in intensive care unit patients with increased bleeding risk and obesity. A prospective analysis of 1000 procedures. Acta Anaesthesiol Scand. 2011;55(7):835-841.

3. Ullman J, Karling J, Margolin G. A new safe and cost-effective percutaneous dilatational tracheotomy: SafeTrach. Acta Otolaryngol. 2016;136(6):598-605.

4. Margolin G, Ullman J, Karling J. A new Technique for percutaneous tracheotomy. Otolaryngol Head Neck Surg. 2017;156(5):966-968. 